\title{
Metodología para modelizar una red de tráfico en la que se van a obtener datos mediante la técnica del escaneo de matrículas
}

\author{
Santos Sánchez-Cambronero \\ Ana Rivas \\ Rosa M. Barba \\ Lidia Ruiz-Ripoll \\ Inmaculada Gallego \\ José María Menéndez \\ Departamento de Ingeniería Civil y de la Edificación, Universidad de Castilla-La Mancha, \\ España
}

\section{RESUMEN}

En el presente artículo se aborda el problema de modelizar una red de tráfico para poder aplicar la técnica del escaneo de matrículas para estimar flujos en ruta, y por tanto obtener la matriz Origen-Destino así como la asignación de la red. Para llevar a cabo dicha modelización se plantea una metodología que trata de manera global la simplificación de la red y que tiene como base la reducción del número de rutas mediante la eliminación de pares Origen-Destino que no tengan una demanda relevante. Dicha simplificación tiene un enfoque práctico muy diferente de la visión tradicional de zonificación y disposición de centroides dentro de la red y que permitirá imbricarla con los modelos de ubicación de dispositivos de escaneo.

La metodología permite detectar aquellos arcos de la red que son afectados por la simplificación y las consecuencias sobre la estimación de flujos que puedan derivarse de dicha afección. Con todo ello, se puede establecer una priorización en la ubicación de los equipos de escaneo que permitirá hacer una reconstrucción más fiable de los flujos de la red.

Se ha empleado una red basada en la denominada red Nguyen-Dupuis como ejemplo de aplicación de la metodología desarrollada. A través del mismo se irá aclarando paso por paso cada una de las fases del método.

\section{INTRODUCCIÓN}

Uno de los objetivos de los modelos de tráfico es la estimación de una matriz OrigenDestino de calidad que permita a su vez calcular los flujos en las calles de una ciudad. Numerosos autores han tratado de estimar dicha matriz. Willumsen (1978) y Yang et al. (1990) afirmo que los viajes siguen un patrón, proponiendo un modelo calibrado a partir del conteo de flujo de vehículos; Brenninger-Göthe y Jornstern (1998) dieron un paso más reconociendo el error que se podía cometer en la toma de datos, aceptando que no podían 
obtener los flujos de la red con exactitud y aspirando a cumplir solamente una serie de objetivos. Bianco et al. (2001) plantean un modelo de ubicación de sensores que permita obtener dichos flujos. El número y la ubicación de los dispositivos fueron también estudiados por autores como Yim y Lam (1998), Yang y Zhou (1998) o Ehlert et al. (2005). Por otro lado, Castillo et al. (2008 a, b) proponen el uso de Redes Bayesianas como herramienta tanto para calcular una matriz O-D como para ubicar los dispositivos de aforo.

Castillo et al. (2008 c) plantean la obtención de la matriz Origen-Destino y la reconstrucción de los flujos de rutas basándose en los datos obtenidos mediante el escaneo de matrículas en los arcos de la red, gracias a la elección óptima de aquellos arcos que han de ser escaneados para obtener los mencionados flujos. A pesar de disponer de la localización de arcos que han de ser escaneados para tener todos los datos de flujo, no siempre será posible ubicar equipos en dichos arcos. Mínguez et al. (2010) abordan el problema del emplazamiento óptimo de equipos bajo unas determinadas condiciones o restricciones como puede ser un presupuesto limitado para la adquisición de dispositivos de escaneo o una determinada fiabilidad de los resultados.

Si bien se ha estudiado donde ubicar las cámaras para el escaneo en base a una determinada red y teniendo en cuenta un determinado presupuesto o fiabilidad deseada, en el presente artículo se aborda el problema desde una perspectiva global, teniendo en cuenta la red y que permite además localizar y acotar la afección sobre los resultados que se deriva de la modelización del caso de estudio. Hasta ahora, los modelos propuestos no han considerado la forma de obtener la red, lo que puede ser un problema debido a dos motivos principalmente:

- Si se considera la red completa, sin llevar a cabo ninguna simplificación, esta puede ser muy grande, contener muchas rutas, etc. Esto trae consigo una gran complejidad a la hora de obtener los flujos de tráfico debido al alto coste computacional que ello acarrea.

- Por otro lado, si la red está excesivamente simplificada pueden aparecer muchos errores al estimar los flujos.

La modelización de la red es un problema que ha sido abordado durante décadas, pero siempre pensando en redes en las que se iban a aplicar métodos convencionales en los que sus datos de partida eran aforos de tráfico. Para llevar a cabo dicha modelización, de manera tradicional, se ha recurrido al uso de centroides. Como recoge Sheffi (1985), cada uno de estos centroides agrega todos los orígenes y/o destinos de la zona en la que está contenido el mismo. Mediante conectores, dichos centroides se conectan al resto de la red. El empleo de centroides y conectores lleva asociado un error en la estimación de flujos en base a la elección de los mismos, Mann (2002) plantean la división en sub-zonas de cada una de las zonas iniciales para eliminar la dependencia de los resultados respecto a la ubicación de centroides y conectores. Por otro lado, Quian y Zhang (2012) estudian la 
selección y ubicación óptima de los mismos teniendo en cuenta las implicaciones que tiene dicha selección.

Ahora bien, esta forma de simplificar y abordar el problema no es no es la más adecuada para el empleo de la técnica del escaneo de matrículas para la obtención de flujos, pues centroide y conector resultan ser conceptos abstractos que se han venido utilizando de manera teórica pero que, más allá del entendimiento general de la red, carecen de sentido a la hora de pasar de la teoría a la práctica.

Es por ello que, en el presente artículo se desarrollará una metodología para modelizar la red acotando los posibles efectos derivados y reduciendo el coste computacional del problema a resolver. Tener una red simplificada implica que será necesario un menor número de equipos para escanear los flujos esperados. Además como se explicará en el apartado correspondiente a la metodología, es posible saber en qué arcos posiblemente se obtendrán estimaciones erróneas al hacer la simplificación, así como una aproximación de la magnitud del error. De esta forma, una vez obtenido el modelo de tráfico, se podrá abordar una reconstrucción de la red que se adapte a las condiciones de fiabilidad y presupuesto impuestas a priori, y que permita obtener datos de flujos de tráfico mediante el escaneo de matrículas.

A continuación se va a desarrollar la metodología planteada para simplificar la red y se hará a través de un ejemplo, que trata de facilitar la comprensión de la misma. Como consecuencia de la aplicación del método desarrollado se han extraído una serie de conclusiones que se exponen al final del artículo.

\section{METODOLOGÍA}

En este apartado se explica la metodología propuesta. La base del proceso a seguir está en la reducción del número de rutas mediante la supresión de la condición de origen y/o destino en algunos nodos, si estos cumplen una condición preestablecida.

\subsection{Datos e hipótesis de partida}

\subsubsection{Definición de la red}

El punto de partida es una definición lo más exhaustiva posible de la red, que incluya todos los nodos y rutas posibles. Para facilitar la comprensión de la metodología, se va a explicar ésta a través de un ejemplo. Se va a emplear una red que está basada en la denominada "red de Nguyen-Dupuis", cuyas características se exponen en la Figura 1: 


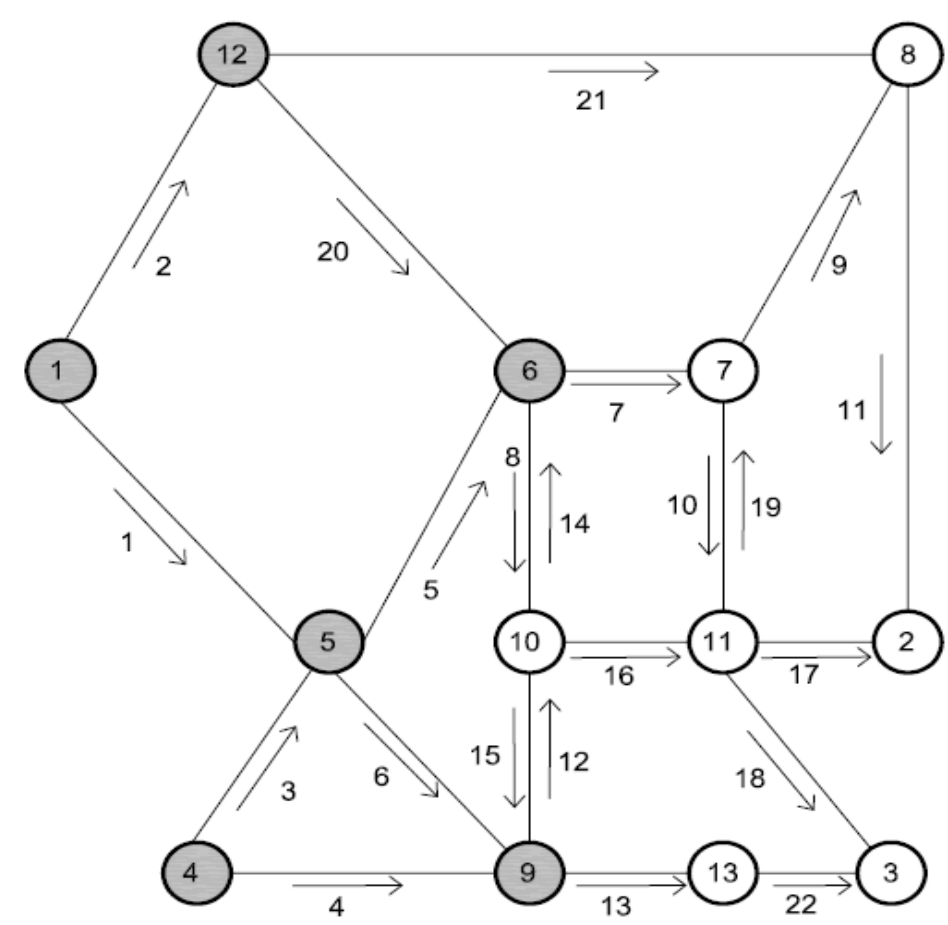

Fig. 1 - Esquema de la red, basada en la propuesta por Nguyen-Dupuis, empleada en el ejemplo de aplicación de la metodología

Se trata de una red con $22 \operatorname{arcos}$ y 13 nodos, seis de ellos serán considerados orígenes (sombreados en la Figura 1) $\{1,4,5,6,9,12\}$ y el resto serán destinos $\{2,3,7,8,10,11$, 13\}. A continuación se definen las diferentes tipologías de nodo existentes dentro de la red:

- Nodo Origen: se trata de un nodo generador. Se ha considerado que será el primero atravesado por el vehículo desde el inicio de su viaje.

- Nodo Destino: se trata de un nodo atractor. Se ha hecho la hipótesis de que será aquel nodo atravesado por última vez por el vehículo antes de finalizar su viaje.

- Nodo de Paso: no es generador ni atractor de viajes y por tanto no habrá rutas que se originen o terminen en él.

Como se dijo en la Introducción, se plantea una alternativa a la zonificación tradicional y el uso de centroides. Dicha alternativa reside en la definición de Nodo Origen y Destino que se ha hecho anteriormente. Supongamos que el esquema de la Figura 2 representa una zona de una red urbana. Tradicionalmente, todos los viajes que se iniciasen o finalizasen dentro de la zona, lo harían en el centroide "C" (Sheffi (1985)). Dicho centroide es un punto que no se encuentra físicamente en la red y que ha sido ubicado por el analista en base a diversas consideraciones, que no tienen que ser de naturaleza física sino que a menudo atienden a otros factores (Quian y Zhang (2012)). La hipótesis que se plantea en este trabajo es que todos los vehículos que atraviesen el nodo "N1" por primera vez, tengan a éste como nodo origen, como es el caso del vehículo "A". En el caso de los nodos destinos, vehículos como el "B" cuyo último nodo atravesado en su ruta es el "N2", tendrán a éste 
como nodo destino. Tanto "N1" como "N2", son puntos existentes dentro de la red (cruces de calles) lo que da una visión práctica y realista que facilita la posterior ubicación de equipos de escaneo sobre la red.

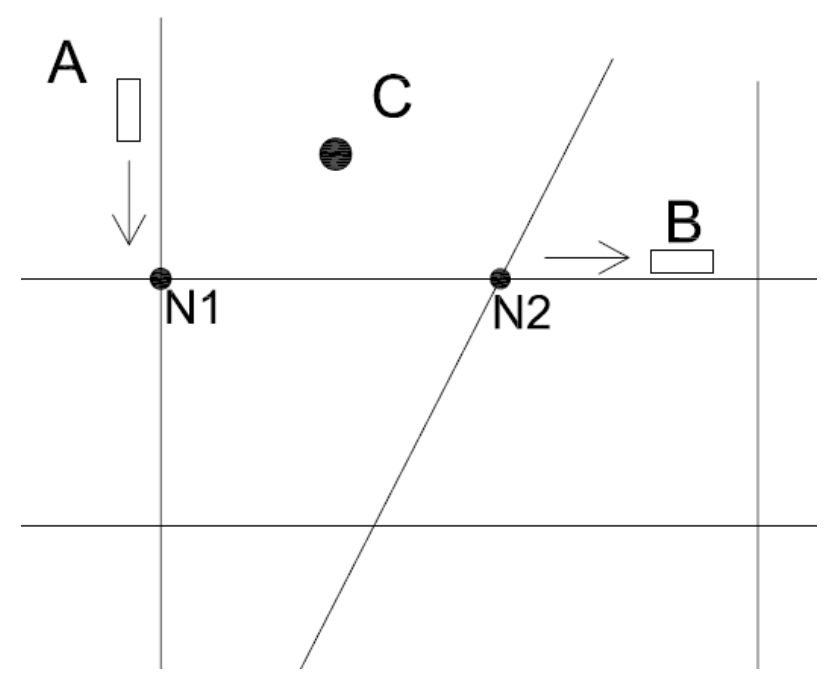

Fig. 2 - Esquema de una red tipo. Comparación perspectiva tradicional (zonificación y centroides) y propuesta definición Nodos Origen y Nodos Destino

Cabe aclarar, que cualquier nodo podrá tener condición de origen, destino o nodo de paso, en función de la ruta que se esté evaluando.

Para finalizar con la definición de la red, en la Tabla 1, se puede comprobar para cada arco $a$, el coste asociado cuando no hay flujo $\left(\mathrm{c}_{\mathrm{a}}\right)$.

\subsubsection{Demanda de referencia}

Además de disponer de una red exhaustiva, es necesario partir de una demanda de referencia que servirá para tomar decisiones sobre qué elementos de la red simplificar. En la Tabla 2 se muestra dicha demanda para el caso del ejemplo. Se muestran en filas los orígenes y en columnas los destinos, apareciendo en la última columna (en negrita) los viajes generados $G i$ en cada nodo origen, mientras que en la última fila se aprecia (en negrita) los viajes atraídos $A j$ por cada nodo destino. Existen un total de 20 pares OrigenDestino.

Una vez que se dispone de la demanda de referencia entre los pares origen-destino existentes, el primer paso será enumerar las rutas que aparecerán dentro de la red y asignar la proporción de demanda correspondiente a cada una de ellas. Para ello se puede utilizar cualquier método de asignación; en nuestro caso se ha empleado el método propuesto por Wardrop (1952). Aparece así el conjunto de rutas originales $\{\mathrm{R}\}$ (en la Tabla 3 se muestran por nodos) y la demanda de referencia de cada una de ellas. 


\begin{tabular}{clc}
\hline Id Arco & Arco & $\mathrm{c}_{\mathrm{a}}$ \\
\hline 1 & $1-5$ & 7 \\
2 & $1-12$ & 9 \\
3 & $4-5$ & 12 \\
4 & $4-9$ & 5 \\
5 & $5-6$ & 12 \\
6 & $5-9$ & 9 \\
7 & $6-7$ & 5 \\
8 & $6-10$ & 4 \\
9 & $7-8$ & 9 \\
10 & $7-11$ & 8 \\
11 & $8-2$ & 9 \\
12 & $9-10$ & 5 \\
13 & $9-13$ & 9 \\
14 & $10-6$ & 4 \\
15 & $10-9$ & 4 \\
16 & $10-11$ & 4 \\
17 & $11-2$ & 9 \\
18 & $11-3$ & 8 \\
19 & $11-7$ & 4 \\
20 & $12-6$ & 7 \\
21 & $12-8$ & 14 \\
22 & $13-3$ & 11 \\
\hline
\end{tabular}

Tabla 1 -Definición de arcos y coste con flujo nulo para cada arco

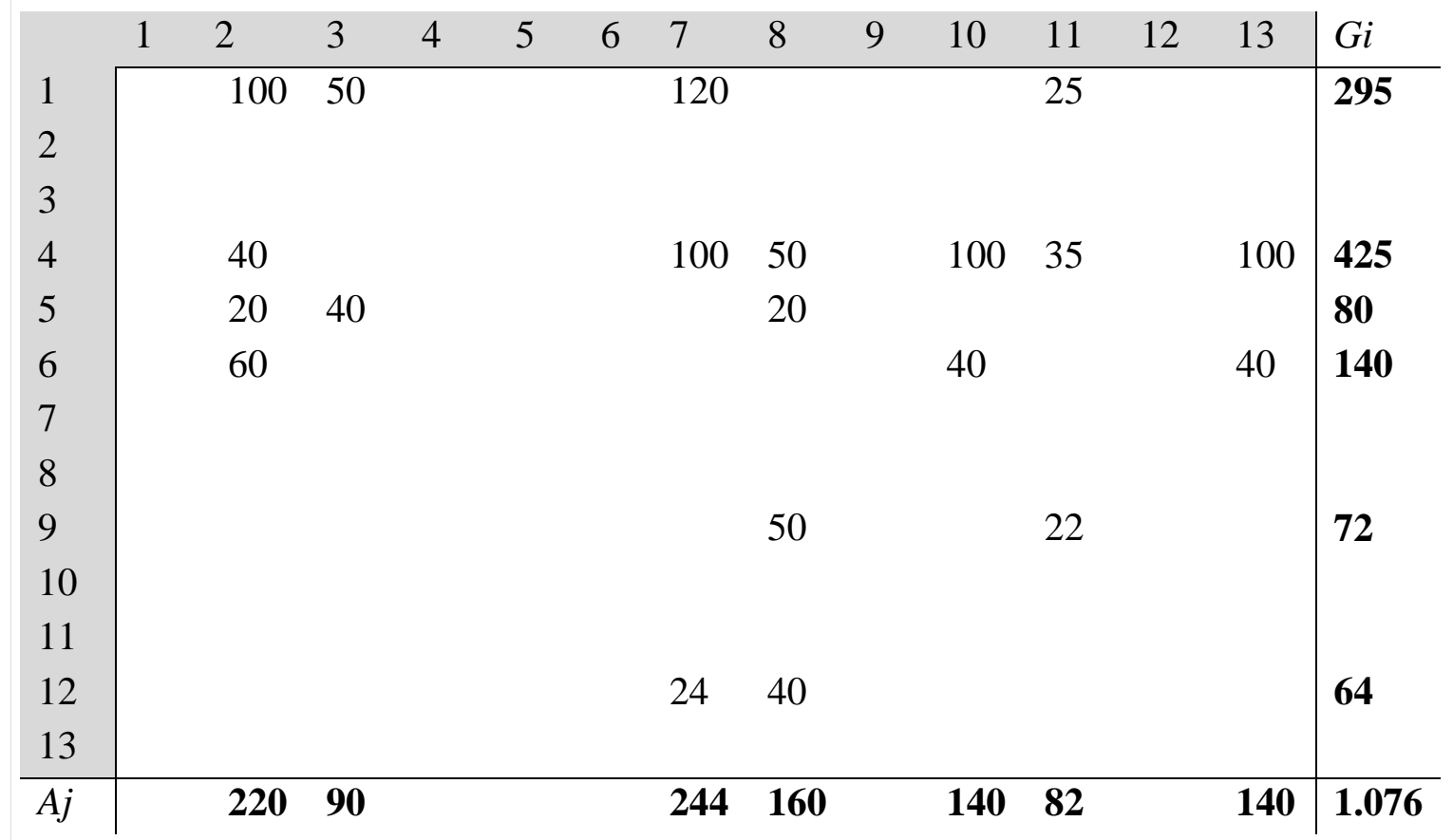

Tabla 2 -Matriz Origen Destino de la red original 


\begin{tabular}{|c|c|c|c|c|c|c|c|}
\hline Id & 1 & 2 & 3 & 4 & 5 & 6 & Demanda $(F)$ \\
\hline $\mathbf{R 1}$ & 1 & 12 & 8 & 2 & & & 100 \\
\hline $\mathbf{R} 2$ & 1 & 5 & 6 & 7 & 11 & 3 & 9 \\
\hline $\mathbf{R 3}$ & 1 & 5 & 6 & 10 & 11 & 3 & 19 \\
\hline R4 & 1 & 5 & 9 & 13 & 3 & & 15 \\
\hline R5 & 1 & 12 & 6 & 7 & 11 & 3 & 7 \\
\hline R6 & 1 & 5 & 6 & 7 & & & 34 \\
\hline R7 & 1 & 12 & 6 & 7 & & & 86 \\
\hline R8 & 1 & 5 & 6 & 7 & 11 & & 21 \\
\hline R9 & 1 & 5 & 6 & 10 & 11 & & 4 \\
\hline R10 & 4 & 9 & 10 & 11 & 2 & & 40 \\
\hline R11 & 4 & 5 & 6 & 7 & & & 79 \\
\hline R12 & 4 & 9 & 10 & 11 & 7 & & 21 \\
\hline R13 & 4 & 9 & 10 & 11 & 7 & 8 & 50 \\
\hline R14 & 4 & 9 & 10 & & & & 100 \\
\hline R15 & 4 & 9 & 10 & 11 & & & 35 \\
\hline R16 & 4 & 9 & 13 & & & & 100 \\
\hline R17 & 5 & 6 & 10 & 11 & 2 & & 20 \\
\hline R18 & 5 & 6 & 10 & 11 & 3 & & 26 \\
\hline R19 & 5 & 9 & 13 & 3 & & & 14 \\
\hline R20 & 5 & 6 & 7 & 8 & & & 20 \\
\hline R21 & 6 & 7 & 11 & 2 & & & 9 \\
\hline R22 & 6 & 10 & 11 & 2 & & & 51 \\
\hline R23 & 6 & 10 & & & & & 40 \\
\hline R24 & 6 & 10 & 9 & 13 & & & 40 \\
\hline R25 & 9 & 10 & 6 & 7 & 8 & & 50 \\
\hline R26 & 9 & 10 & 11 & & & & 22 \\
\hline R27 & 12 & 6 & 7 & & & & 24 \\
\hline \multirow[t]{2}{*}{ R28 } & 12 & 8 & & & & & 40 \\
\hline & & & & & & & 1.076 \\
\hline
\end{tabular}

Tabla 3 -Conjunto de rutas $\{R\}$ asociadas a la red original

\subsubsection{Criterio para abordar la simplificación de la red}

Como ya se ha mencionado, la base de la metodología reside en la supresión de la condición de nodo origen y/o destino en algunos nodos. Para que se pueda eliminar dicha condición se ha de cumplir el siguiente criterio: La demanda generada o atraída por el nodo ha de ser menor a un umbral establecido por el analista. Para el caso del ejemplo se ha considerado una demanda mínima (generada o atraída) de 100 vehículos.

\subsection{Procedimiento}

El proceso a seguir será iterativo y estará estructurado en 3 pasos, como se ve en la Figura 3. Dicho proceso se seguirá tanto para el caso de nodos origen como para el de nodos destino, y se parará cuando tras ir evaluando nodos, no exista ninguno que cumpla el criterio de simplificación. 


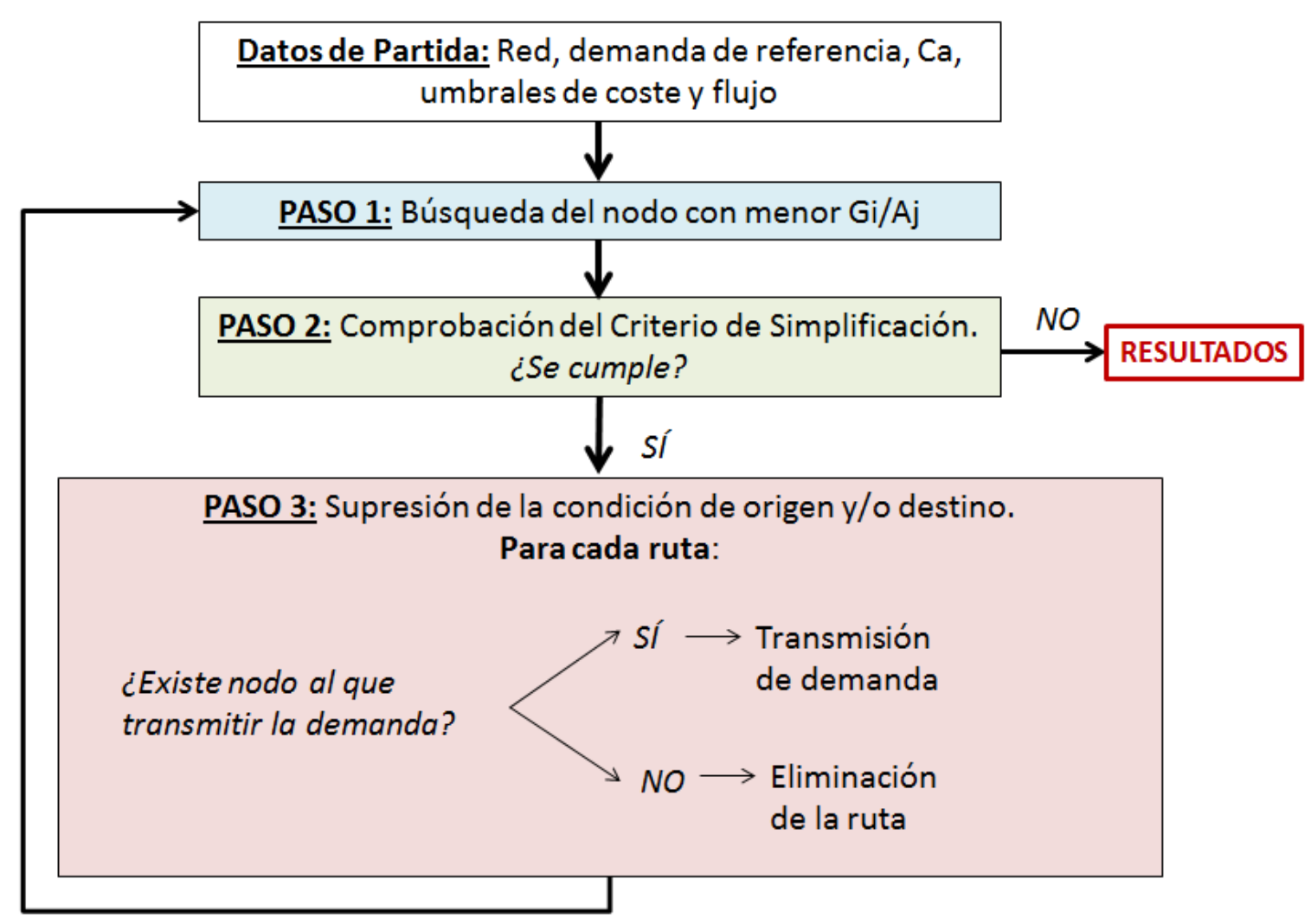

Fig. 3 - Esquema de la metodología propuesta

\subsubsection{PASO 1: Búsqueda del nodo con menor demanda}

El "criterio de simplificación" hace referencia a que los nodos que perderán su condición de origen y/o destino han de estar por debajo de una demanda mínima, es por ello que se buscará en primer lugar el nodo con la menor demanda, que será el candidato a perder su condición de origen/destino.

Para el caso del ejemplo, si se retoma la Tabla 2, se puede comprobar que en este caso el nodo que se empezaría a evaluar es el 12 , que se trata de un nodo origen y que tiene un flujo de referencia generado de 64.

\subsubsection{PASO 2: Comprobación del criterio de simplificación}

Cuando se dispone de los nodos candidatos a perder su condición, es necesario comprobar que se cumple el "criterio de simplificación", es decir, se comprobará que la demanda está por debajo del umbral; si no lo estuviese, se daría por finalizado el proceso.

Si se retoma el ejemplo, el nodo 12, que era candidato a ser eliminado como origen, tiene una demanda de 64. Puesto que se había establecido el umbral en 100, el nodo 12 puede eliminarse en el siguiente paso del proceso.

\subsubsection{PASO 3: Supresión de la condición de origen y/o destino}

Una vez comprobado el cumplimiento del “criterio de simplificación”, el nodo perdería su 
condición de origen y/o destino. Esto implica que ninguna ruta podrá empezar o acabar en el nodo en cuestión. Por lo tanto, será necesario transmitir el flujo de dichas rutas a los nodos del entorno, siempre que esto sea posible.

Dicha transmisión de flujo se hará evaluando cada una de las rutas implicadas (las rutas que empiecen o acaben, según el caso, en el nodo evaluado) y solo se llevará a cabo si se cumple el siguiente "criterio de transmisión": se ha de poder transmitir la demanda del nodo al que se le ha quitado su condición a otro nodo de la misma naturaleza del entorno. La transmisión se hará así mismo siempre y cuando el nodo al que se le va a ceder la demanda esté a una distancia, a través de la ruta, por debajo de un umbral. Dicha distancia se evaluará teniendo en cuenta el coste cuando no hay flujo ( $c_{a}$ ) (ver Tabla 1) en los arcos involucrados. Para el caso del ejemplo se ha considerado un coste máximo de 15.

De no poder transmitir el flujo a ningún nodo del entorno, la ruta en cuestión así como la demanda asociada a la misma se perderían en el modelo.

Según se vaya produciendo la transmisión de flujos, se irán rehaciendo las rutas, apareciendo un nuevo conjunto simplificado $\{Q\}$, que se actualizará en cada una de las iteraciones del proceso. En el caso de nodos destinos, se procedería de forma análoga.

Para aclarar este paso, se retoma el nodo 12 en el ejemplo. Dos rutas salen desde dicho nodo (ver Tabla 4):

Como se ha dicho, se evalúan todas las rutas que se inicien en el "nodo suprimido", en este caso el 12. Para la ruta R27, el nodo aguas abajo del 12 es el 6, que es origen. Además el coste del arco existente entre ambos nodos es 7 que está por debajo del umbral. Por lo tanto para esta ruta se podría transmitir la demanda de la misma desde el nodo 12 al nodo 6 .

Si se evalúa la ruta R28, el nodo aguas abajo del nodo 12 es el nodo 8, que tiene condición de destino. Por lo tanto el nodo 12 no podría transmitir su demanda en este caso, como se ha enunciado antes, la ruta y la demanda asociada a la misma se perderían (ver Tabla 4):

\begin{tabular}{ccccccc|c} 
Id & 1 & 2 & 3 & 4 & 5 & 6 & Demanda $(\mathrm{F})$ \\
\hline $\mathbf{R 2 7}$ & 6 & 7 & & & & & 24 \\
R28 & 12 & 8 & & &
\end{tabular}

Tabla 4 -Aplicación del paso 3 de la metodología a las rutas que tenían originalmente al nodo 12 como origen

El nodo 12 se suprimiría en la primera iteración realizada con los nodos origen. En la segunda se evaluaría el nodo 9 (que tiene una demanda de referencia de 72, ver Tabla 2). $\mathrm{Si}$ se tienen en cuenta las rutas que empiezan en el mismo (ver Tabla 5), aparecen dos 
situaciones:

- En la ruta R25 el flujo se transmitiría al nodo origen más cercano que es el nodo 6 (aparece marcado en la Tabla 5). Como se puede comprobar, existe un nodo más anterior, el 10, pero este tiene inicialmente condición de nodo destino (ver Figura 1). Transmitir el flujo del nodo 9 al 6 tiene un coste de 9, menor al umbral por lo tanto se cumple el "criterio de transmisión"

- En la ruta R26, no existe ningún nodo origen aguas abajo del nodo 9, por lo tanto se perdería la ruta y el flujo asociado a la misma, como se aprecia en la Tabla 5.

\begin{tabular}{ccccccc|c} 
Id & 1 & 2 & 3 & 4 & 5 & 6 & Demanda $(\mathrm{F})$ \\
\hline $\mathbf{R 2 5}$ & 6 & 7 & 8 & & & 50 \\
$\mathbf{R 2 6}$ & 9 & 10 & 11 & &
\end{tabular}

Tabla 5 -Aplicación del paso 3 de la metodología a las rutas que tenían originalmente al nodo 9 como origen

En el caso del ejemplo, tras hacer todas las iteraciones posibles, se llega a que los nodos origen que perderán su condición como tal son $\{5,9,12\}$ y en el caso de los destinos $\{3$, $11\}$.

\subsection{Resultados}

\subsubsection{Conjunto simplificado de rutas $\{Q\}$}

Una vez realizadas todas las iteraciones posibles, se llega al conjunto simplificado de rutas por nodos $\{\mathrm{Q}\}$ correspondiente a la red modelizada que se muestra en la Tabla 6 junto con la demanda de referencia asociada.

Como se puede comprobar, se ha reducido el número de rutas respecto a la red original. Además ninguna de las existentes dentro del conjunto $\{Q\}$ empieza o termina en los nodos que han perdió su condición de origen y/o destino. Por otro lado, si se compara la demanda total de la red original (ver Tabla 3) con la demanda total de la red simplificada (ver Tabla 6), se puede comprobar que ha disminuido. Esto es debido a la supresión de algunas rutas, que se hacía durante el Paso 3 del procedimiento, y la pérdida de flujo que ello conlleva.

\subsubsection{Flujo en arco}

A partir de los resultados de la red simplificada, en términos de rutas y flujo en ruta, se puede obtener el flujo de referencia en cada uno de los arcos:

$$
V_{a}=\sum_{r} \delta_{r}^{a} F_{r}
$$




\begin{tabular}{|c|c|c|c|c|c|c|c|}
\hline Id & 1 & 2 & 3 & 4 & 5 & 6 & Demanda $(F)$ \\
\hline Q1 & 1 & 12 & 8 & 2 & & & 100 \\
\hline Q2 & 1 & 5 & 6 & 10 & & & 23 \\
\hline Q3 & 1 & 5 & 9 & 13 & & & 15 \\
\hline Q4 & 1 & 12 & 6 & 7 & & & 86 \\
\hline Q5 & 1 & 5 & 6 & 7 & & & 55 \\
\hline Q6 & 4 & 9 & 10 & 11 & 2 & & 40 \\
\hline Q7 & 4 & 5 & 6 & 7 & & & 79 \\
\hline Q8 & 4 & 9 & 10 & 11 & 7 & & 21 \\
\hline Q9 & 4 & 9 & 10 & 11 & 7 & 8 & 50 \\
\hline Q10 & 4 & 9 & 10 & & & & 135 \\
\hline Q11 & 4 & 9 & 13 & & & & 100 \\
\hline Q12 & 6 & 10 & 11 & 2 & & & 71 \\
\hline Q13 & 6 & 10 & & & & & 66 \\
\hline Q14 & 6 & 7 & 8 & & & & 70 \\
\hline Q15 & 6 & 7 & 11 & 2 & & & 9 \\
\hline Q16 & 6 & 10 & 9 & 13 & & & 40 \\
\hline Q17 & 6 & 7 & & & & & 24 \\
\hline
\end{tabular}

Tabla 6 -Conjunto de rutas $\{Q\}$ asociadas a la red modelizada

Donde $V_{a}$ es el flujo en el $\operatorname{arco} a, \mathrm{y} \delta_{r}^{a}$ es 1 si el $\operatorname{arco} a$ pertenece a la ruta $r$ y 0 en caso contrario, $F_{r}$ es el flujo en la ruta $\mathrm{r}$. Si obtenemos el flujo en arco de la red original (primera columna Tabla 7) y se compara con el flujo en arco de la red simplificada (segunda columna Tabla 7), se puede detectar qué arcos son susceptibles de estimar su flujo de manera indirecta (ver cuarta columna de la Tabla 7) al simplificar la red, y de que magnitud es este.

\section{CONCLUSIONES Y FUTURAS LÍNEAS DE INVESTIGACIÓN}

A continuación se exponen algunas conclusiones que se han extraído del desarrollo de la metodología y de la aplicación de la misma a la red del ejemplo:

- Los arcos a escanear propuestos serán los correspondientes a la red simplificada. Como es de esperar, será un menor número que los necesarios en la red original. Disminuirá por tanto el presupuesto, aunque esto implicará una menor precisión en los resultados.

- Se intuye que se cometerá un error en la estimación de los flujos de aquellos arcos implicados en la simplificación de la red. Dicho error estará acotado.

- Resulta interesante retomar la información recabada en la Tabla 7. Es recomendable priorizar la ubicación de equipos de escaneo en aquellos arcos en los que se espera cometer un error mayor, de manera que este se detectase mediante el escaneo, pudiendo actuar frente a él y subsanarlo parcial o totalmente en fases sucesivas del 
análisis. Este aspecto de la investigación está aún por concluir y será objeto de estudio de futuros trabajos.

- Dicha priorización se puede hacer en base al porcentaje de error que se espera cometer (ver columna 4 de la Tabla 7), de manera que se detecten aquellos errores de mayor magnitud.

\begin{tabular}{c|cccc} 
Arco (a) & Va & Va* $^{*}$ & Diferencia & \% Error \\
\hline 1 & 102 & 93 & 9 & 8,82 \\
2 & 193 & 186 & 7 & 3,63 \\
3 & 79 & 79 & 0 & 0 \\
4 & 346 & 346 & 0 & 0 \\
5 & 232 & 157 & 75 & 32,33 \\
6 & 29 & 15 & 14 & 48,28 \\
7 & 339 & 323 & 16 & 4,72 \\
8 & 200 & 200 & 0 & 0 \\
9 & 120 & 120 & 0 & 0 \\
10 & 46 & 9 & 37 & 70,43 \\
11 & 100 & 100 & 0 & 0 \\
12 & 318 & 246 & 72 & 22,64 \\
13 & 169 & 155 & 14 & 8,28 \\
14 & 50 & 0 & 50 & 100 \\
15 & 40 & 40 & 0 & 0 \\
16 & 288 & 182 & 106 & 36,81 \\
17 & 120 & 120 & 0 & 0 \\
18 & 61 & 0 & 61 & 100 \\
19 & 71 & 71 & 0 & 0 \\
20 & 117 & 86 & 31 & 26,50 \\
21 & 140 & 100 & 40 & 28,57 \\
22 & 29 & 0 & 29 & 100
\end{tabular}

Tabla 7-Comparativa de flujo en arco en la red original y la red modelizada. Magnitud del error cometido

\section{REFERENCIAS}

WILLUMSEN, L. G. (1978). Estimation of od matriz from traffic counts. A review working, paper 99. Institute of Transportation Studies, University of Leeds.

YANG, H., IIDIA, Y., SASAKI, T., 1991. An analysis of the reliability of an origindestination trip matrix estimated from traffic counts. Transportation Research Part B 25(5), pp. 351-363.

BIANCO, L., CONFESSORE, L., REVERBERI, O., 2001. A network based model for traffic sensor location with implications on O/D matrix estimates. Transportation Science 35(1), pp. 50-60.

BRENNINGER-GOTHE, M. JORNSTERN, K. (1989). Estimation of origin-destination matrices from traffic counts using multiobjective programming formulations. Transportation Research, 23B, pp. 257-269

CASTILlO, E., MENÉNDEZ, J.M., JIMÉNEZ, P., 2008b. Trip matrix and path flow reconstruction and estimation based on plate scanning and link observations. 
Transportation Research Part B 42(5), pp. 455-481.

MÍNGUEZ, R., SÁNCHEZ-CAMBRONERO, S., CASTILLO, E., JIMÉNEZ, P., 2010. Optimal traffic plate scanning location for OD trip matrix and route estimation in road networks. Transportation Research Part B 44(2), pp. 282-298.

YIM, P. K. N., LAM, W. H. K. (1998). Evaluation of count location selection methods for estimation of od matrices. Journal of Transportation Engineering, ASCE, pp. 376-383.

YANG, H., ZHOU, J. (1998). Optimal traffic counting locations for origin-destination matrix estimation. Transportation Research, 32B, pp. 109-126

EHLERT, A., BELL, M.G.H., GROSSO, S. (2006). The optimization of traffic count locations in road networks. Transportation Research, 40B, pp. 460-479.

CASTILLO, E., MENÉNDEZ, J.M., SÁNCHEZ-CAMBRONERO, S., 2008 a. Predicting traffic flow using Bayesian Networks. Transportation Research Part B, 42(5), pp. 482509.

CASTILlO, E., MENÉNDEZ, J.M., SÁNCHEZ-CAMBRONERO, S., 2008 b. Traffic estimation and optimal counting location without path enumeration using Bayesian Networks. Computer-Aided Civil and Infraestructure Engineering, 23(3), pp. 189-207.

CASTILlO, E., MENÉNDEZ, J.M., SÁNCHEZ-CAMBRONERO, S., 2008 c. Traffic estimation and optimal counting location without path enumeration using Bayesian networks. Computer Aided Civil and Infraestructure Engineering, 23, 2 Special issue on traffic computational models.

SHEFFI, Y., 1985. Urban Transportation Networks. Equilibrium Analysis with Mathematical Programming Methods. Prentice-Hall Inc.

MANN, W.W., 2002. B-node model: new subarea traffic assignment model and application. Eighth TRB Conference on the Application of Transportation Planning Methods.

QUIAN, Z.S., ZHANG, H.M., 2012. On centroid connectors in static traffic assignment: their effects on flow patterns and how to optimize their selections. Transportation Research Part B 46, pp. 1489-1503.

WARDROP, J.G., 1952. Some Theoretical Aspects of Road Traffic Research. Road Engineering Division Meeting 24 January 1952, Road Paper No. 36 